\title{
Raising research quality will require collective action
}

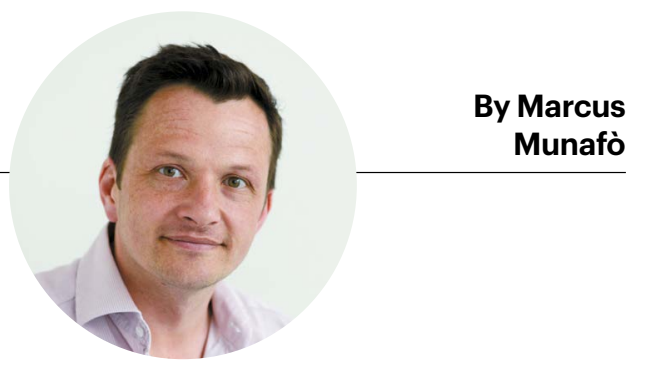

\section{Institutions must act together to reform research culture, says Marcus Munafò.}

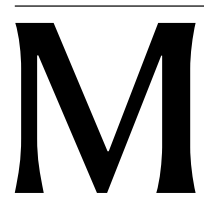

odern research is far removed from that done by the independent scientist of Charles Darwin's day. But that model still underpins what we reward. And the tension is showing. The number of authors listed on published papers has increased fivefold. But our system of incentives - accreted haphazardly as science evolved from hobby to career - still focuses on individuals.

Funding, appointments, promotions, tenure, prizes and so on emphasize individual achievement and overlook deeds that benefit everyone and should be valued explicitly, such as producing usable tools or sharing code. If we want to move towards a transparent model of research, we need to reward open-research practices. If we want researchers to work well in large collaborations, we need to train them in communication skills and collective self-scrutiny.

In the past five years, many studies have attempted to assess the prevalence of questionable research practices. The Netherlands has commissioned a country-wide survey. Others have focused on specific fields, from ecology and evolution to health services. Describing the scale of the problem is necessary, but insufficient.

We must reflect on how (and, more importantly, why) questionable research practices and undesirable behaviours arise and persist. What are the flaws in our institutions' cultures and practices that allow this conduct to proliferate? We must find the root causes. Have we disincentivized solid, cumulative work, replication studies and publishing null results - essential if science is to self-correct efficiently - with our relentless focus on groundbreaking findings?

That self-inspection is in the air. Institutions are committing to working together to determine how their cultural practices, such as emphasizing the importance of novelty, discovery and priority, undermine the value of replication, verification and transparency. That is the goal of the UK Reproducibility Network, which I co-founded earlier this year.It started as informal groups of researchers at individual institutions that met with representatives from funders and publishers (including Nature) who were open to discussions about how best to align open-science initiatives - reproducibility sections in grant applications and reporting checklists in article submissions, for example. Now institutions themselves are cooperating to consider larger changes, from training to hiring and promotion practices.

Our ten university members span the United Kingdom from Aberdeen to Surrey, and we expect that list to grow. Each will appoint a senior academic to focus on research quality and improvement. Figuring out which system-level

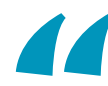

If we want

to move

towards a

transparent model of research, we need to reward openresearch practices."

Marcus Munafò is professor of biological psychology at the University of Bristol, UK, and chair of the UK Reproducibility Network Steering Group. e-mail: marcus. munafo@bristol. ac.uk changes are needed and how to make them happen will now be someone's primary responsibility, not a volunteer activity. What changes might ensue? Earlier this year, the University of Bristol, where I work, made the use of data sharing and other open-research practices an explicit criterion for promotion.

But one institution will make little difference on its own. For better practices to become the norm, many universities need to act collectively. Changes to incentives at a single institution will not make new behaviours stick, not least because practices required in only one place can act as a career tax on its scientists. Only if changes occur across many institutions will the impacts permeate scientific culture.

The same is true for training. If universities agree that all their graduates must reach common standards - in data skills, for example - future scientists will do better research and collaborate across institutions in a way that is hard to imagine today. Consider the productivity gains if competence in the programming language $R$ - which can be used to run statistical analyses across a broad range of areas - was a given. Research would become more efficient, like the railways did after adopting a common standard for track gauge.

Public-health campaigns to reduce smoking demonstrate how coordinated action can yield broad cultural change. In $1974,45 \%$ of the UK population smoked; now it is below $15 \%$. This was not the result of any one intervention, but a coordinated programme of taxes, bans in the workplace and public spaces, and advertising restrictions. Gradually, the 'normal' behaviour changed. Someone wishing to smoke today would probably ask their companions for permission; a generation ago, they would have offered them a cigarette.

When it comes to changing the culture of science, the UK Reproducibility Network is not alone. Numerous initiatives now link members of the research community to support robust, transparent research. Examples include the Center for Open Science in the United States (founded in 2013), the QUEST Center in Germany (founded in 2017), the Research on Research Institute with eight participating countries, launched this year, and a proliferation of grassroots networks of researchers in many countries.

But these cultural changes might falter. Culture eats strategy for breakfast - grand plans founder on the rocks of implicit values, beliefs and ways of working. Top-down initiatives from funders and publishers will fizzle out if they are not implemented by researchers, who review papers and grant proposals. Grass-roots efforts will flourish only if institutions recognize and reward researchers' efforts.

Funders, publishers and bottom-up networks of researchers have all made strides. Institutions are, in many ways, the final piece of the jigsaw. Universities are already investing in cutting-edge technology and embarking on ambitious infrastructure programmes. Cultural change is just as essential to long-term success. 